\title{
REVISITING THE BLACK HOLE MASSES OF SOFT X-RAY-SELECTED ACTIVE GALACTIC NUCLEI
}

\author{
Linda C. Watson, ${ }^{1}$ Smita Mathur, ${ }^{1}$ and Dirk Grupe ${ }^{2}$ \\ Received 2006 November 20; accepted 2007 February 15
}

\begin{abstract}
In our previous work, using luminosity and the $\mathrm{H} \beta \mathrm{FWHM}$ as surrogates for black hole mass $\left(M_{\mathrm{BH}}\right)$, we compared the black hole masses of narrow-line Seyfert 1 galaxies (NLS1s) and broad-line Seyfert 1 galaxies (BLS1s) in a sample of soft X-ray-selected active galactic nuclei. We found that the distributions of black hole masses in the two populations are statistically different. Recent work shows that the second moment of the $\mathrm{H} \beta$ emission line (the line dispersion) is a better estimator of black hole mass than FWHM. To test whether changing the width measure affects our results, we calculate line dispersion-based black hole masses for our soft X-ray-selected sample. We find that using the line dispersion rather than the FWHM as a measure of the gas velocity shifts NLS1 and BLS1 virial product distributions closer together, but they remain distinct. On the $M_{\mathrm{BH}}-\sigma_{*}$ plane, we find that using the line dispersion leaves NLS1s below the $M_{\mathrm{BH}}-\sigma_{*}$ relation, but to a less significant degree than when FWHM is used to calculate black hole masses (the [O III] $25007 \mathrm{FWHM}$ is used as a surrogate for the bulge stellar velocity dispersion). The level of significance of our findings is such that we cannot draw firm conclusions on the location of the two samples on the $M_{\mathrm{BH}^{-}} \sigma_{*}$ plane. We are still left with two alternative scenarios: either (1) NLS1s lie below the $M_{\mathrm{BH}^{-}} \sigma_{*}$ relation, indicating that their black hole masses are growing, or (2) NLS1s lie on the $M_{\mathrm{BH}}-\sigma_{*}$ relation, so they preferentially reside in smaller mass, less luminous galaxies; the present data do not allow us to choose one over the other. More trustworthy stellar velocity dispersions and accurate black hole mass measurements with reverberation mapping are required for a firmer statement about the locus of NLS1s on the $M_{\mathrm{BH}^{-}} \sigma_{*}$ plane.
\end{abstract}

Key words: galaxies: active — galaxies: nuclei — quasars: general

\section{INTRODUCTION}

In the study of the coevolution of host galaxies and their central black holes, one of the most interesting and useful tools is the firm correlation between black hole mass $\left(M_{\mathrm{BH}}\right)$ and stellar velocity dispersion of the host bulge $\left(\sigma_{*}\right)$. The $M_{\mathrm{BH}}-\sigma_{*}$ relation was first established for quiescent galaxies, where black hole masses were mainly determined using gas and stellar dynamics (Ferrarese \& Merritt 2000; Gebhardt et al. 2000a) and in a few cases with maser kinematics (e.g., NGC 4258; Miyoshi et al. 1995) and proper motion (Galactic center; Genzel et al. 2000; Ghez et al. 2000). With further study, the $M_{\mathrm{BH}}-\sigma_{*}$ relation was found to extend to active galaxies, where traditional mass measurement techniques are no longer feasible because the region directly affected by the black hole is unresolved (Gebhardt et al. 2000b; Ferrarese et al. 2001). For type 1 active galactic nuclei (AGNs), the most direct technique for measuring black hole masses is reverberation mapping (Blandford \& McKee 1982; Peterson 1993). Here the time delay between continuum and associated emission-line variations is used with the width of the emission line to calculate a virial mass. However, reverberation mapping is time-intensive, and thus a common practice in determining black hole masses is to employ an empirical relation between the radius of the broad-line region $\left(R_{\mathrm{BLR}}\right)$ and the monochromatic continuum luminosity $\left(L_{5100}\right)$, as derived in, e.g., Kaspi et al. (2000) or Bentz et al. (2006). The $R_{\mathrm{BLR}}-L_{5100}$ relation is calibrated against reverberation-mapped AGNs and allows one to calculate $R_{\mathrm{BLR}}$ and therefore the virial mass using a singleepoch observation. Consequently, black hole masses can be estimated for large samples of AGNs.

\footnotetext{
${ }^{1}$ Department of Astronomy, The Ohio State University, Columbus, OH 43210, USA; watson@astronomy.ohio-state.edu,smita@astronomy.ohio-state.edu.

2 Department of Astronomy and Astrophysics, Pennsylvania State University, University Park, PA 16802, USA; grupe@astro.psu.edu.
}

This contribution continues the three-paper series investigating the locus of narrow-line Seyfert 1 galaxies (NLS1s) on the $M_{\mathrm{BH}}-\sigma_{*}$ plane (Grupe \& Mathur 2004; Mathur \& Grupe 2005a, 2005b). NLS1s are defined as those AGNs having an $\mathrm{H} \beta$ emission line FWHM $\leq 2000 \mathrm{~km} \mathrm{~s}^{-1}$ (Osterbrock \& Pogge 1985). The comparatively low emission-line widths of NLS1s are commonly accepted as evidence for a low-mass black hole powering the AGN and often go hand-in-hand with a high accretion rate and a steep soft X-ray slope (Pounds et al. 1995; Grupe et al. 1998). NLS1s are therefore a focal point for extreme AGN physical properties and also occupy a unique and interesting position on the $M_{\mathrm{BH}}-\sigma_{*}$ relation.

Grupe \& Mathur (2004) studied a sample of 75 soft X-rayselected Seyfert 1 galaxies, including 32 NLS1s and 43 broad-line Seyfert 1 galaxies (BLS1s), extending the earlier work of Mathur et al. (2001). Using the $\mathrm{H} \beta$ FWHM and the Kaspi et al. (2000) $R_{\mathrm{BLR}}-L_{5100}$ relation to estimate the black hole mass and the width of the [O III] $\lambda 5007$ emission line $\left(\sigma_{[\mathrm{O}} \mathrm{III}\right)$ as a surrogate for the stellar velocity dispersion, they found that NLS1s as a class lie below the $M_{\mathrm{BH}}-\sigma_{*}$ relation. Furthering the study, Mathur \& Grupe (2005a) distinguished between those NLS1s well below the $M_{\mathrm{BH}}-\sigma_{*}$ relation and those near the $M_{\mathrm{BH}}-\sigma_{*}$ relation and found that those NLS1s that lie below the $M_{\mathrm{BH}}-\sigma_{*}$ relation also have larger Eddington ratios $\left(L_{\mathrm{bol}} / L_{\mathrm{Edd}}\right)$ and steeper soft X-ray slopes compared to those NLS1s that lie near the $M_{\mathrm{BH}}{ }^{-} \sigma_{*}$ relation. Both results led the authors to conclude that highly accreting AGNs at low redshift lie below the $M_{\mathrm{BH}}-\sigma_{*}$ relation, while AGNs with low accretion rates lie close to the $M_{\mathrm{BH}}-\sigma_{*}$ relation, having achieved their final black hole mass.

In Grupe \& Mathur (2004) neither the black hole masses based on $\mathrm{H} \beta \mathrm{FWHM}$ nor the stellar velocity dispersions based on $\left.\sigma_{[\mathrm{O}} \mathrm{mI}\right]$ are direct measurements. Therefore, the most prudent approach is to test that $\mathrm{H} \beta \mathrm{FWHM}$ and $\sigma_{[\mathrm{O} \mathrm{m}]}$ accurately describe $M_{\mathrm{BH}}$ and $\sigma_{*}$, respectively. By far the most suspect of the two estimations is 
substituting $\sigma_{[\mathrm{O} \text { II] }}$ for $\sigma_{*}$ (Boroson 2003; Greene \& Ho 2005). Consequently, Mathur \& Grupe (2005b) address this point by focusing on concerns put forth by Greene \& $\mathrm{Ho}(2005)$ that $\sigma_{[\mathrm{O}}$ III $]$ is not only a function of $\sigma_{*}$ but also of $L_{\text {bol }} / L_{\text {Edd }}$. Correcting for this dependence does not change the results of Grupe \& Mathur (2004); while no individual object's location on their $\left.M_{\mathrm{BH}}-\sigma_{[\mathrm{O}} \mathrm{III}\right]$ plot should necessarily be trusted, the overall result is sound in that highly accreting NLS1s lie below the $M_{\mathrm{BH}}-\sigma_{*}$ relation (Mathur \& Grupe 2005b).

In this work we address the $M_{\mathrm{BH}}$ estimates for the soft X-rayselected sample of Grupe \& Mathur (2004). The motivations for this study are the recent publications by Peterson et al. (2004) and Collin et al. (2006). Our discussion is based on black hole masses $\left(M_{\mathrm{BH}}\right)$ calculated using

$$
M_{\mathrm{BH}}=f \frac{R_{\mathrm{BLR}}(\Delta V)^{2}}{G},
$$

where $f$ is a scale factor that depends on the geometry and kinematics of the BLR, $R_{\mathrm{BLR}}$ is the radius of the BLR, and $\Delta V$ is a measure of the BLR gas velocity. We often refer to the virial product $\left[\mathrm{VP}=R_{\mathrm{BLR}}(\Delta V)^{2} / G\right]$, which only differs from $M_{\mathrm{BH}}$ by the dimensionless scale factor $f$, which is expected to be of order unity. Peterson et al. (2004) measured the emission-line widths and the time delays between continuum and line variations $(\tau)$ for various emission lines in four reverberation-mapped AGNs. They found that using the second moment of the $\mathrm{H} \beta$ emission line, referred to as the "line dispersion" or $\sigma_{\text {line }}$, as a measure of $\Delta V$ reproduces a $\Delta V \propto \tau^{-1 / 2}$ relation with higher precision than FWHM. They therefore conclude that the line dispersion is a more robust width measure than FWHM because it provides a more constant virial product over multiple observations of an AGN.

In Collin et al. (2006) the authors divided their sample of 14 AGNs in two ways: first, with Population 1 having $\mathrm{H} \beta \mathrm{FWHM} /$ $\sigma_{\text {line }}<2.35$ and Population 2 having FWHM $/ \sigma_{\text {line }}>2.35$, and second, with Population A having $\mathrm{H} \beta \mathrm{FWHM}<4000 \mathrm{~km} \mathrm{~s}^{-1}$ and Population B having FWHM $>4000 \mathrm{~km} \mathrm{~s}^{-1}$. Generally, Populations 1 and $\mathrm{A}$ are considered narrow-line objects, and Populations 2 and $\mathrm{B}$ are considered broad-line objects. They then calculated virial products using both FWHM and $\sigma_{\text {line }}$ as $\Delta V$. To determine the statistical value of the scale factor $f$ for each of the four populations above, they shifted these virial products onto the quiescent galaxy $M_{\mathrm{BH}}-\sigma_{*}$ relation of Tremaine et al. (2002) (this is the method of Onken et al. 2004). When using the H $\beta$ FWHM as $\Delta V$, the scale factors they derive for narrow-line objects and broad-line objects are significantly different. On the other hand, the scale factors computed using the $\mathrm{H} \beta$ line dispersion are consistent with a constant value. Collin et al. (2006) therefore conclude that the line dispersion is less sensitive to whatever property establishes a difference between narrow and broad populations in the eyes of FWHM and is therefore a less biased width measure. The authors provide their best estimates for these various scale factors; the one of most interest for this work is $f=3.85$, which was derived to convert line dispersion-based virial products measured on the mean spectrum into black hole masses.

We begin this work by comparing NLS1 and BLS1 virial product distributions. When we require masses, we adhere to the approach of Grupe \& Mathur (2004) by examining the positions of NLS1s and BLS1s with respect to the $M_{\mathrm{BH}}-\sigma_{*}$ relation under the assumption that a single scale factor is appropriate for both populations. In $\S 2$ we discuss our data analysis technique, detailing our line dispersion measurements. Section 3 describes our results, comparing NLS1 and BLS1 virial products and $M_{\mathrm{BH}}-\sigma_{*}$ distributions when the $\mathrm{H} \beta \mathrm{FWHM}$ or line dispersion is used for $\Delta V$. Finally, we discuss our conclusions in $\S 4$.

\section{DATA ANALYSIS}

Grupe \& Mathur (2004) calculated black hole masses for 75 soft X-ray-selected AGNs (32 NLS1s and 43 BLS1s) using equation (1). For $f$ they used the Kaspi et al. (2000) value of 0.75 , for $\Delta V$ they used the $\mathrm{H} \beta \mathrm{FWHM}$, and for $R_{\mathrm{BLR}}$ they used the Kaspi et al. (2000) $R_{\mathrm{BLR}}-L_{5100}$ relation. Motivated by Peterson et al. (2004) and Collin et al. (2006), in this work we use the same sample but calculate virial products using the $\mathrm{H} \beta$ line dispersion rather than FWHM. We also update our $R_{\mathrm{BLR}}-L_{5100}$ calculation by employing the more recent Bentz et al. (2006) relation.

We carried out measurements on the same $\mathrm{H} \beta$ narrow component- and Fe II-subtracted spectra as in Grupe et al. (2004) and Grupe \& Mathur (2004). We removed four AGNs (one NLS1 and three BLS1s) from our sample because $\mathrm{H} \beta$ was possibly contaminated by an optically thin, very broad $\mathrm{H} \beta$ component (Shields et al. 1995), residual Fe II, or He II. For consistency with Collin et al. (2006) and to avoid a blending conflict with the [O III] $\lambda \lambda 4959$, 5007 lines and $\mathrm{Fe}$ II, we chose to measure the line dispersion using the blue side of the emission line, thus assuming a symmetric line profile. We then measured the $\mathrm{H} \beta$ line dispersion and FWHM for each AGN. We found general agreement between our FWHM measurements and the Grupe \& Mathur (2004) FWHM measurements, signaling that our line dispersion values can also be trusted. We calculated virial products based on these line dispersion measurements and compared them to virial products based on the 2004 FWHM measurements.

Following the procedure of Grupe \& Mathur (2004), we used the width of the [O III] $\lambda 5007$ emission line as a surrogate for the stellar velocity dispersion. We used twice the half-width at halfmaximum (HWHM) of the red side of the [O III] emission line rather than the FWHM to avoid the blue asymmetry discussed in Grupe \& Mathur (2004). Then, $\sigma_{[\mathrm{O} \text { II] }}=2(\mathrm{HWHM} / 2.35)$. We do not present stellar velocity dispersions with the Greene \& Ho (2005) correction to $\sigma_{[\mathrm{O} \text { III }]}$ applied because it does not significantly affect the results (Mathur \& Grupe 2005b).

\section{RESULTS}

\subsection{Virial Product Distributions}

In all the figures in this paper, we have used the Bentz et al. (2006) $R_{\mathrm{BLR}}-L_{5100}$ relation to calculate the $R_{\mathrm{BLR}}$ of the virial product. But, for ease of comparison with the results of Grupe \& Mathur (2004), we quote results using the Kaspi et al. (2000) $R_{\mathrm{BLR}}-L_{5100}$ relation as well.

Also for comparison purposes, we present figures and calculations where $\Delta V$ of equation (1) is the $\mathrm{H} \beta$ FWHM of Grupe \& Mathur (2004) alongside figures where $\Delta V$ is the $\mathrm{H} \beta$ line dispersion. In the FWHM scheme, the top left panel of Figure 1 shows virial product histograms for NLS1s (solid line) and BLS1s (dotted line). The conclusion that these distributions are dissimilar is emphasized by the virial product cumulative fraction plot in the bottom left panel of Figure 1. The Kolmogorov-Smirnov (K-S) test probability that our NLS1 and BLS1 FWHM-based virial products are drawn from the same parent population is $\sim 10^{-9}$ (when the Kaspi et al. [2000] $R_{\mathrm{BLR}}-L_{5100}$ relation is used, the probability is $\sim 10^{-8}$ ).

Now, using our $\mathrm{H} \beta$ line dispersion measurements for $\Delta V$ of equation (1), the top right panel of Figure 1 shows virial product histograms for NLS1s and BLS1s. The bottom right panel of Figure 1 shows the virial product cumulative fraction plot. By 


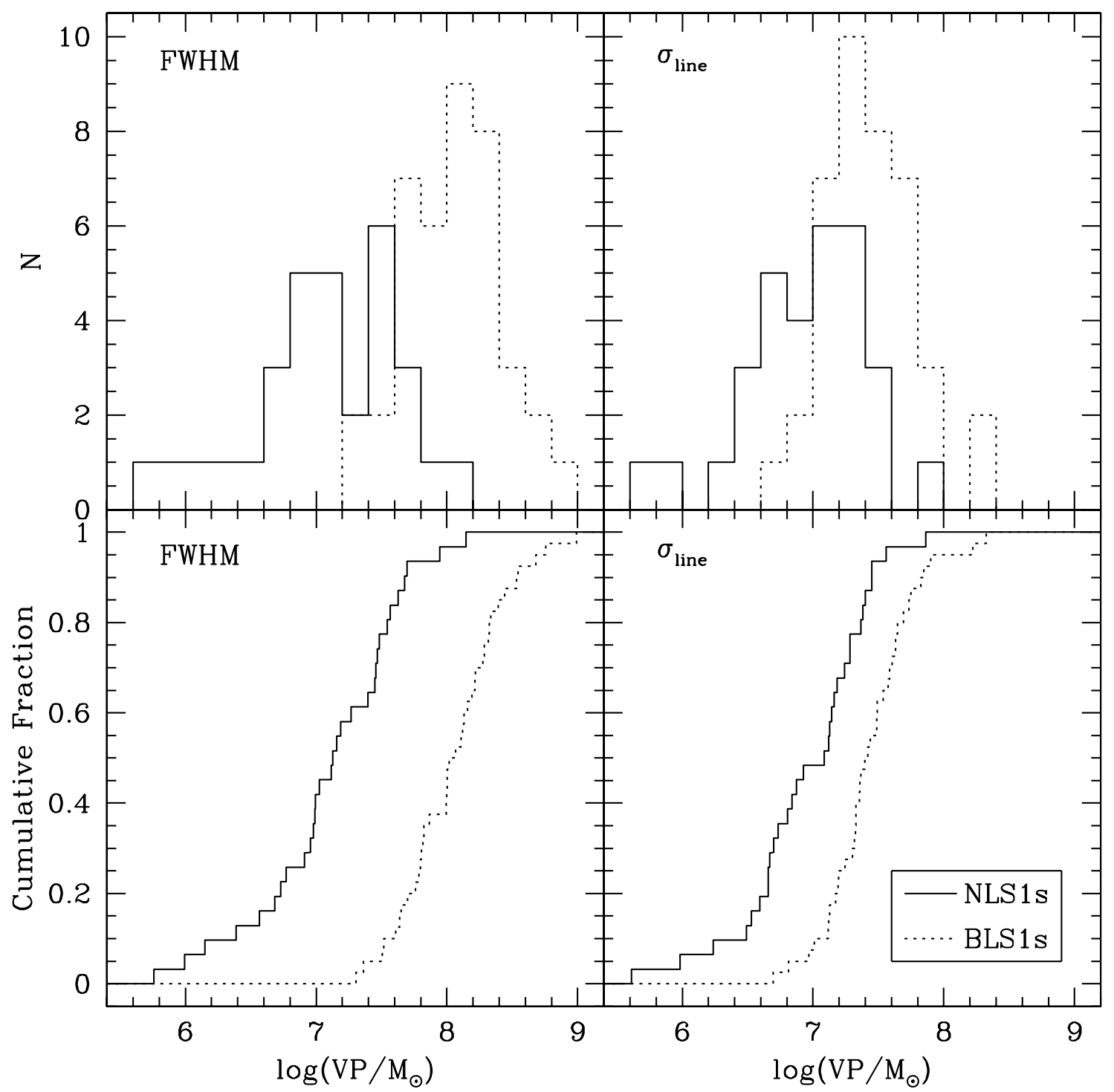

FIG. 1.-Top panels: Virial product histograms for NLS1s (solid lines) and BLS1s (dotted lines). Bottom panels: Cumulative fractions of a K-S test comparing NLS1 and BLS1 virial product distributions. In the left panels we calculate the virial product using the $\mathrm{H} \beta \mathrm{FWHM}$ for $\Delta V$ of eq. (1). In the right panels we calculate the virial product using the $\mathrm{H} \beta$ line dispersion ( $\sigma_{\text {line }}$ ) for $\Delta V$. In all panels of this figure and in all subsequent figures, we use the Bentz et al. (2006) radius-luminosity relation to calculate the $R_{\mathrm{BLR}}$ of eq. (1).

comparing the histograms and cumulative fraction plots of Figure 1, we see that using the line dispersion closes the gap between NLS1 and BLS1 virial product distributions. When the line dispersion is used for $\Delta V$, the probability that our NLS1 and BLS1 samples are drawn from the same virial product population is $\sim 10^{-4}$ (when the Kaspi et al. [2000] $R_{\mathrm{BLR}}-L_{5100}$ relation is used, the probability is 0.004$)$. The large increase in the probability shows that the NLS1 and BLS1 virial product distributions are more similar when one uses the line dispersion rather than the FWHM as the $\mathrm{H} \beta$ width measure. However, the two classes remain significantly different even when the line dispersion is used and NLS1s remain with systematically smaller virial products than BLS1s.

\subsection{Consequences on the $M_{\mathrm{BH}^{-}} \sigma_{*}$ Plane}

The only two viable ways for NLS1s and BLS1s to both lie on the $M_{\mathrm{BH}}-\sigma_{*}$ relation are (1) for NLS1s and BLS1s to have the same black hole mass distributions and the same stellar velocity dispersion distributions, or (2) for NLS1s and BLS1s to have dif- ferent black hole mass distributions (with NLS1s having lower black hole masses than BLS1s) and different stellar velocity dispersion distributions (again, presumably with NLS1s having smaller stellar velocity dispersions than BLS1s). We have shown that using the line dispersion as a measure of $\Delta V$ still produces NLS1 and BLS1 virial product distributions that are significantly different. For this argument, we assume that this virial product difference traces the distinctness of the NLS1 and BLS1 black hole mass distributions as well. In addition, Grupe \& Mathur (2004) found that NLS1s and BLS1s show no significant difference in their distributions of stellar velocity dispersions (the K-S test probability that the NLS1 and BLS1 stellar velocity dispersions are drawn from the same parent population is 0.3 ). This conclusion is dependent on the assumption that the width of the [O III $] 25007$ emission line can be used as a reliable stellar velocity dispersion indicator. Since that question is addressed in Mathur \& Grupe (2005b), we assume here that the $[\mathrm{O}$ III $]$ width is a fair estimator of the velocity dispersion in a statistical sense. We are therefore in the situation where the NLS1 and BLS1 black hole mass distributions are 


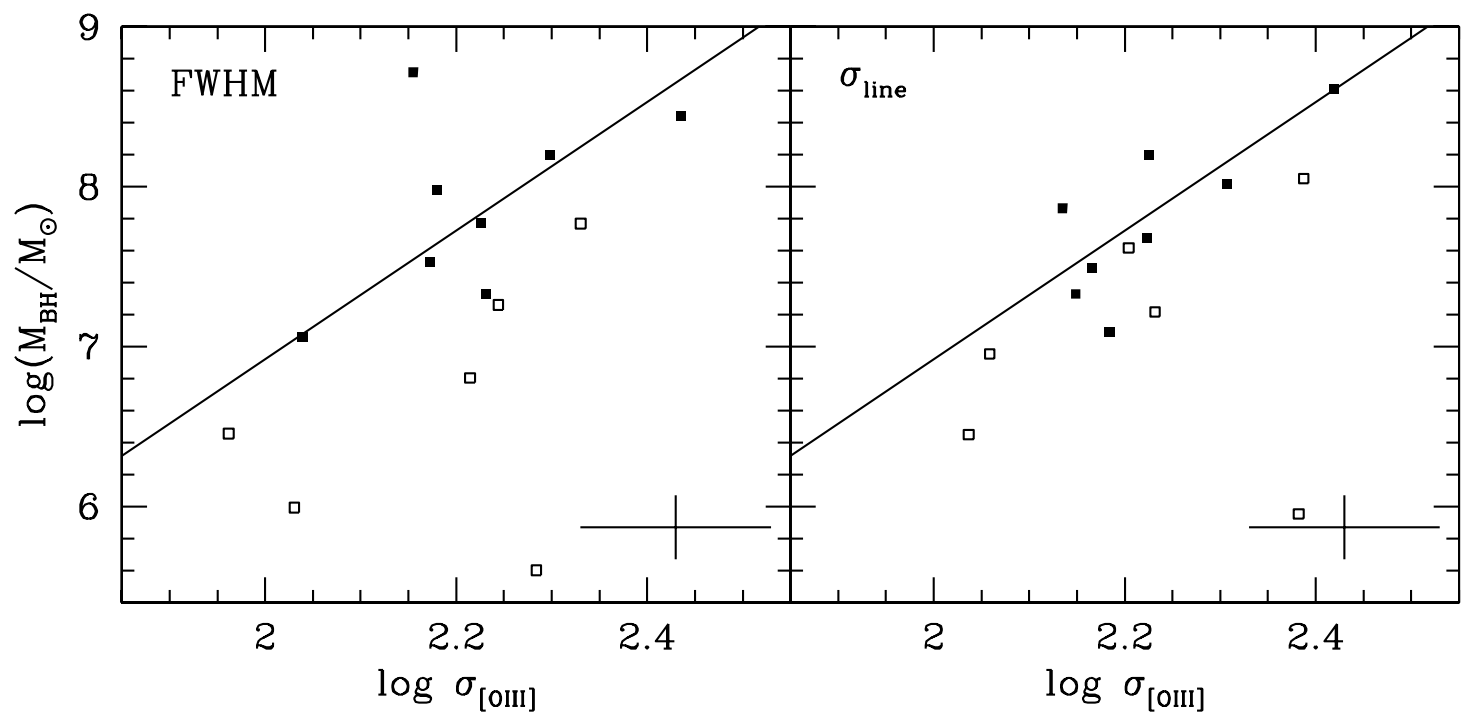

Fig. 2.-Black hole mass vs. stellar velocity dispersion. The squares show the mean $\log \sigma_{[\mathrm{O} \text { ml }}$ for bins in $\log \left(M_{\mathrm{BH}} / M_{\odot}\right)$, with open squares referring to NLS1s and filled squares referring to BLS1s. Here $\left.\sigma_{[\mathrm{O}} \mathrm{ml}\right]$ has units of kilometers per second. The solid lines denote the relation of Tremaine et al. (2002). In the left panel we calculate black hole mass using the $\mathrm{H} \beta \mathrm{FWHM}$ for $\Delta V$ and a scale factor of $f=0.53$. In the right panel we calculate black hole mass using the $\mathrm{H} \beta$ line dispersion and a scale factor of $f=2.19$. In each panel we use the width of the $[\mathrm{O} \mathrm{II}] 25007$ emission line as a surrogate for the stellar velocity dispersion. A typical error bar is shown in the lower right corner of each panel, where we have reduced the error in the original data in accordance with the binning.

significantly different and the stellar velocity dispersion distributions are not significantly different. This implies that, even using the line dispersion as a measure of $\Delta V$, the NLS1 and BLS1 classes should lie at different locations on the $M_{\mathrm{BH}^{-}} \sigma_{*}$ plane.

There are many assumptions in the above argument. We therefore perform two tests comparing the locations of the NLS1s and BLS1s on the $M_{\mathrm{BH}}-\sigma_{*}$ plane. First, we must convert our virial products into black hole masses. We initially used the Kaspi et al. (2000) scale factor of $f=0.75$ to convert our FWHM-based virial products into black hole masses and the Collin et al. (2006) scale factor of $f=3.85$ to convert our line dispersion-based virial products into black hole masses. This procedure left a majority of the BLS1s above both the Tremaine et al. (2002) and the Ferrarese \& Ford (2005) fit to the $M_{\mathrm{BH}}-\sigma_{*}$ relation. In order to test whether the soft X-ray-selected NLS1s lie below the $M_{\mathrm{BH}}-\sigma_{*}$ relation by comparing their location to the soft X-ray-selected BLS1s, we require our sample of BLS1s to be minimally scattered around the $M_{\mathrm{BH}}-\sigma_{*}$ fit. Accordingly, in the remaining analysis we use masses calculated by applying the scale factor that minimizes the rms scatter of the BLS1s around the Tremaine et al. line (this is a modified version of the procedure detailed in Onken et al. 2004). We found the best scale factors to be $f=0.53$ to convert FWHM-based virial products into black hole masses and $f=2.19$ to convert line dispersion-based virial products into black hole masses. We also completed our analysis using the Ferrarese \& Ford (2005) fit to the $M_{\mathrm{BH}}-\sigma_{*}$ relation, where we found $f=0.56$ to be the scale factor that best converts FWHM-based virial products into black hole masses and $f=2.27$ to be the scale factor that best converts line dispersion-based virial products into black hole masses. Because the Tremaine et al. (2002) relation was a better fit to our data, we use it in all relevant figures. We present results based on the Ferrarese \& Ford (2005) relation as well but note that changing the $M_{\mathrm{BH}}-\sigma_{*}$ relation did not significantly affect our results.

Figure 2 compares the locations of BLS1s and NLS1s on the $M_{\mathrm{BH}}-\sigma_{*}$ plane. The filled squares represent BLS1s, the open squares represent NLS1s, and the solid line marks the Tremaine et al. (2002) fit to the $M_{\mathrm{BH}}-\sigma_{*}$ relation. The data have been binned in $\log \left(M_{\mathrm{BH}} / M_{\odot}\right)$, and we have plotted the average value of $\log \left(M_{\mathrm{BH}} / M_{\odot}\right)$ versus the average value of $\log \left(\sigma_{[\mathrm{O} \text { III }}\right)$ for each bin, where $\sigma_{[\mathrm{O} \text { III }]}$ has units of kilometers per second. The left and right panels show the locations of the AGNs on the $M_{\mathrm{BH}}-\sigma_{*}$ plane when black hole masses are calculated using the $\mathrm{H} \beta$ FWHM and the line dispersion, respectively. In the lower right corner of each panel, we show typical error bars. Our calculation of the typical error in $\log \left(M_{\mathrm{BH}} / M_{\odot}\right)$ for an individual object considers the rms scatter in the $R_{\mathrm{BLR}}-L_{5100}$ relation, error in the measurement of the FWHM or the line dispersion, and the unknown geometry of the BLR, which all together amounts to about 0.5 dex. Errors for the [O III] 25007 emission line FWHM are given in Grupe et al. (2004). Based on these values, we give a conservative value of 0.2 dex for the error in $\log \left(\sigma_{[\mathrm{O} \text { III }}\right)$ for an individual object. This error is only the measurement error and therefore does not include any error associated with using the width of [O $\mathrm{III}]$ as a surrogate for the stellar velocity dispersion. The error bars shown in the figure are the individual object values divided by $\sqrt{5}$, where 5 is the average number of AGNs in each bin.

The NLS1s certainly do not appear to lie as pronouncedly below the Tremaine et al. (2002) line when the line dispersion is used to calculate black hole masses. With the large scatter in the unbinned data and the small number of points in the binned data, we chose to use the Mann-Whitney $U$-test on the binned data to determine the probability that the NLS1s and BLS1s are drawn from the same population in their $M_{\mathrm{BH}}$ to $\sigma_{[\mathrm{O}}^{4.02}$. rat ratios. Using the FWHM to calculate black hole masses, we found the probability that the NLS1 and BLS1 samples are drawn from the same population in their $M_{\mathrm{BH}}$ to $\sigma_{[\mathrm{O} \text { II] }}^{4.02}$ ratios to be 0.001 . We also compared the NLS1 and BLS1 $M_{\mathrm{BH}}$ to $\sigma_{[\mathrm{O}}^{4.86}$ rit ratios, where 4.86 is the slope of the Ferrarese \& Ford (2005) $M_{\mathrm{BH}}-\sigma_{*}$ relation; the probability remains 0.001 . Clearly, the NLS1 and BLS1 samples are different in the FWHM case. When the line dispersion is used, the probability that the NLS1s and BLS1s are drawn from the same population in their $M_{\mathrm{BH}}$ to $\sigma_{[\mathrm{O} \text { III }}^{4.02}$ ratios increases to 0.01 , and the probability that they are drawn from the same population in their $M_{\mathrm{BH}}$ to $\sigma_{[\mathrm{O} \text { III] }}$ ratios increases to 0.041 . The exact probability values are sensitive to the binning parameters, but the Mann-Whitney $U$-test shows that there is evidence, albeit less strong than in the 


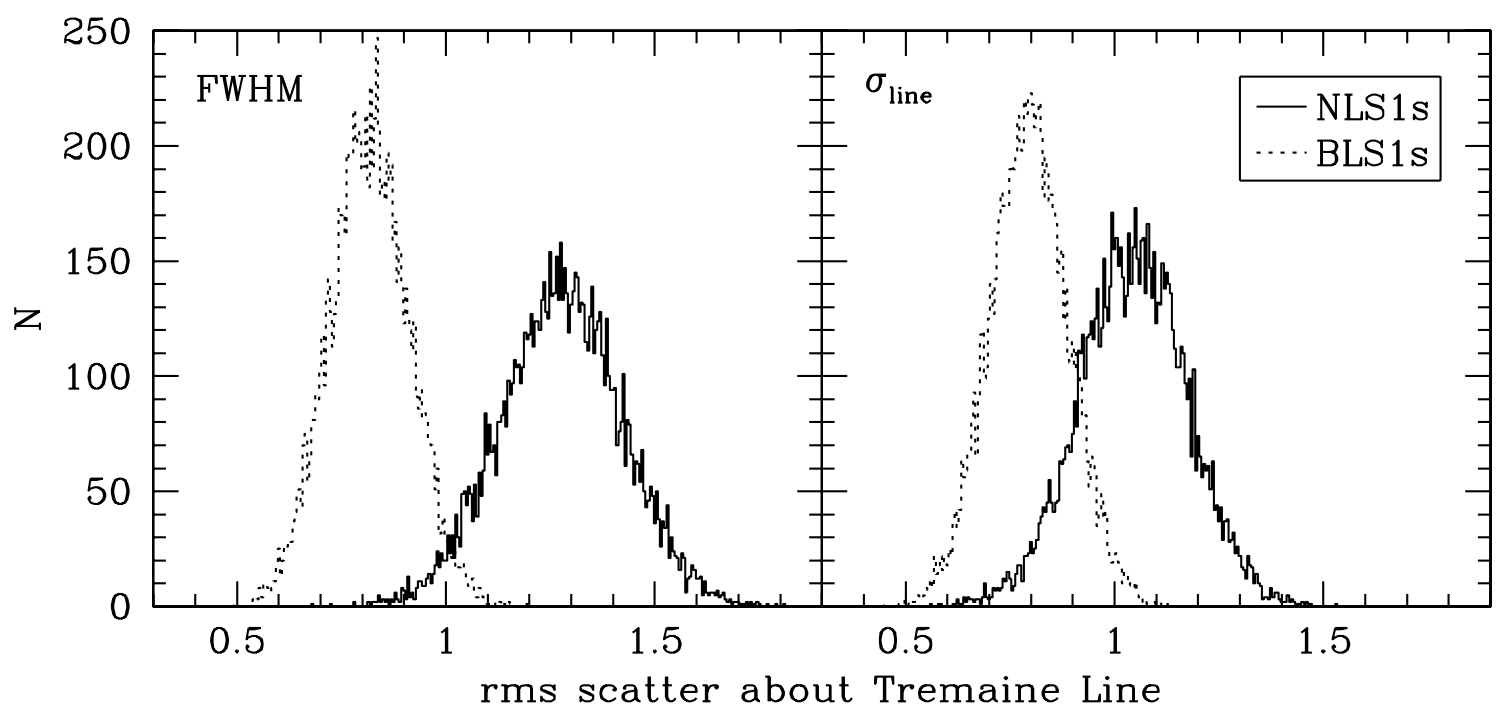

FIG. 3.- Histograms showing the results of a bootstrap analysis on the rms scatter of our sample of NLS1s (solid lines) and BLS1s (dotted lines) around the Tremaine et al.

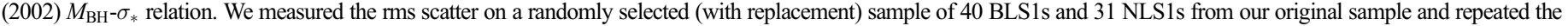

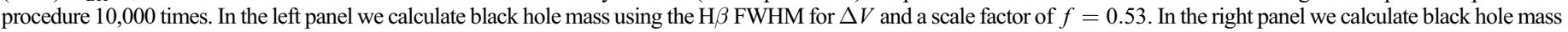
using the $\mathrm{H} \beta$ line dispersion and a scale factor of $f=2.19$. We use the width of the [O III $\lambda 25007$ emission line as a surrogate for the stellar velocity dispersion.

FWHM case, that NLS1s and BLS1s are drawn from different parent populations in their $M_{\mathrm{BH}}$ to $\sigma_{[\mathrm{O} \mathrm{III}]}^{4.02}$ and $M_{\mathrm{BH}}$ to $\sigma_{[\mathrm{O} \text { III }}^{4.8}$ ratios, with NLS1s having systematically smaller values than BLS1s.

We completed one final test comparing the locations of the NLS1s and BLS1s on the $M_{\mathrm{BH}}-\sigma_{*}$ plane. We simply measured the rms scatter of the unbinned data around the Tremaine et al. (2002) line for both the NLS1s and the BLS1s. Using the $\mathrm{H} \beta$ FWHM to calculate black hole masses, the NLS1 rms scatter is $1.29 \mathrm{dex}$ and the BLS1 rms scatter is $0.81 \mathrm{dex}$ in $\log \left(M_{\mathrm{BH}} / M_{\odot}\right)$. Using the $\mathrm{H} \beta$ line dispersion to calculate black hole masses, the NLS1 rms scatter is 1.04 dex and the BLS1 rms scatter is 0.79 dex. While the BLS1 scatter around the Tremaine et al. (2002) line is very similar when the FWHM or the line dispersion is used to calculate black hole masses, the NLS1 scatter is larger in the FWHM case compared to the line dispersion case. In other words, the NLS1s are farther from the Tremaine et al. line in the FWHM case. To test the significance of the rms scatter difference between NLS1s and BLS1s in both the FWHM and line dispersion cases, we used the bootstrap method to estimate an error in each rms value. We used a random number generator (Press et al. 1992, p. 274) to randomly select a sample of 31 NLS1s and 40 BLS1s from our original sample, with replacement. We measured the rms scatter on this new sample and repeated the process 10,000 times. Histograms with the results of these realizations are shown in Figure 3, with the solid line referring to NLS1s and the dotted line referring to BLS1s. The left panel of the figure shows the rms scatter histograms when the $\mathrm{H} \beta$ FWHM is used to calculate black hole masses. Here the average NLS1 rms scatter is $1.28 \mathrm{dex}$ in $\log \left(M_{\mathrm{BH}} / M_{\odot}\right)$, with a standard deviation of $0.15 \mathrm{dex}$. The average BLS1 rms scatter is 0.82 dex, with a standard deviation of 0.10 dex. The right panel shows the rms scatter histograms when the $\mathrm{H} \beta$ line dispersion is used to calculate black hole masses. Here the average NLS1 rms scatter is $1.04 \mathrm{dex}$ in $\log \left(M_{\mathrm{BH}} / M_{\odot}\right)$, with a standard deviation of 0.13 dex. The average BLS1 rms scatter is 0.80 , with a standard deviation of 0.10 dex. The NLS1 and BLS1 average rms scatter values differ by $3.1 \sigma$ in the FWHM case and $1.9 \sigma$ in the line dispersion case (using the NLS1 standard deviation as $\sigma$ ). We also completed the above analysis using the rms scatter around the Ferrarese \& Ford (2005) relation and provide these results in Table 1 . Independent of the $M_{\mathrm{BH}}-\sigma_{*}$ relation used, the results of this rms scatter test are in agreement with the results of the Mann-Whitney $U$-test: NLS1s and BLS1s certainly lie in different locations on the $M_{\mathrm{BH}}-\sigma_{*}$ plane when the FWHM is used to calculate black hole masses. In addition, NLS1s and BLS1s remain in different locations on the $M_{\mathrm{BH}}-\sigma_{*}$ plane when the line dispersion is used to calculate black hole masses, but the difference is less significant.

\subsection{Eddington Ratio Comparison}

Figure 4 shows histograms of $\log \left(L_{\mathrm{bol}} / L_{\mathrm{Edd}}\right)$ for three samples: the soft X-ray-selected NLS1s of this work (solid line), the optically selected NLS1s of Greene \& Ho (2004; dashed line), and the soft X-ray-selected BLS1s of this work (dotted line). One should view this figure with caution, because the black hole masses and the Eddington ratios were calculated differently for the soft X-ray-selected AGNs and the optically selected NLS1s. To calculate the Eddington ratios for the soft X-ray-selected NLS1s and BLS1s, we use the $\mathrm{H} \beta$ line dispersion-based black hole masses. In contrast, Greene \& Ho (2004) use the H $\alpha$ FWHM to calculate black hole masses for the optically selected NLS1s (we have used the corrected masses of Barth et al. 2005). In addition, Greene \&

TABLE 1

The rms Scatter Values

\begin{tabular}{|c|c|c|c|c|c|}
\hline \multirow[b]{2}{*}{$\begin{array}{l}\text { SAMPLE } \\
\text { (1) }\end{array}$} & \multirow{2}{*}{$\begin{array}{c}\text { Galaxy } \\
\text { TyPe } \\
(2)\end{array}$} & \multicolumn{2}{|c|}{ Tremaine } & \multicolumn{2}{|c|}{ FERRARESE } \\
\hline & & $\begin{array}{l}\text { FWHM } \\
\text { (3) }\end{array}$ & $\begin{array}{c}\sigma_{\text {line }} \\
(4)\end{array}$ & $\begin{array}{c}\text { FWHM } \\
\text { (5) }\end{array}$ & $\begin{array}{c}\sigma_{\text {line }} \\
(6)\end{array}$ \\
\hline \multirow[t]{2}{*}{ Original ........ } & NLS1s & 1.29 & 1.04 & 1.41 & 1.21 \\
\hline & BLS1s & 0.81 & 0.79 & 0.96 & 0.95 \\
\hline \multirow[t]{2}{*}{ Bootstrap ..... } & NLS1s & $1.28(0.15)$ & $1.04(0.13)$ & $1.47(0.16)$ & $1.28(0.15)$ \\
\hline & BLS1s & $0.82(0.10)$ & $0.80(0.10)$ & $1.02(0.13)$ & $1.01(0.13)$ \\
\hline
\end{tabular}

Notes.-Comparison of rms scatter values around the Tremaine et al. (2002) and Ferrarese \& Ford (2005) fit to the $M_{\mathrm{BH}}-\sigma_{*}$ relation. Each rms scatter value has units of dex in $\log \left(M_{\mathrm{BH}} / M_{\odot}\right)$. In the first two rows we give the rms scatter values of NLS1s and BLS1s when the FWHM (cols. [3] and [5]) or the line dispersion (cols. [4] and [6]) is used to calculate black hole masses. In the second two rows we provide the average rms scatter values from our bootstrap analysis, with standard deviation values in parentheses. 


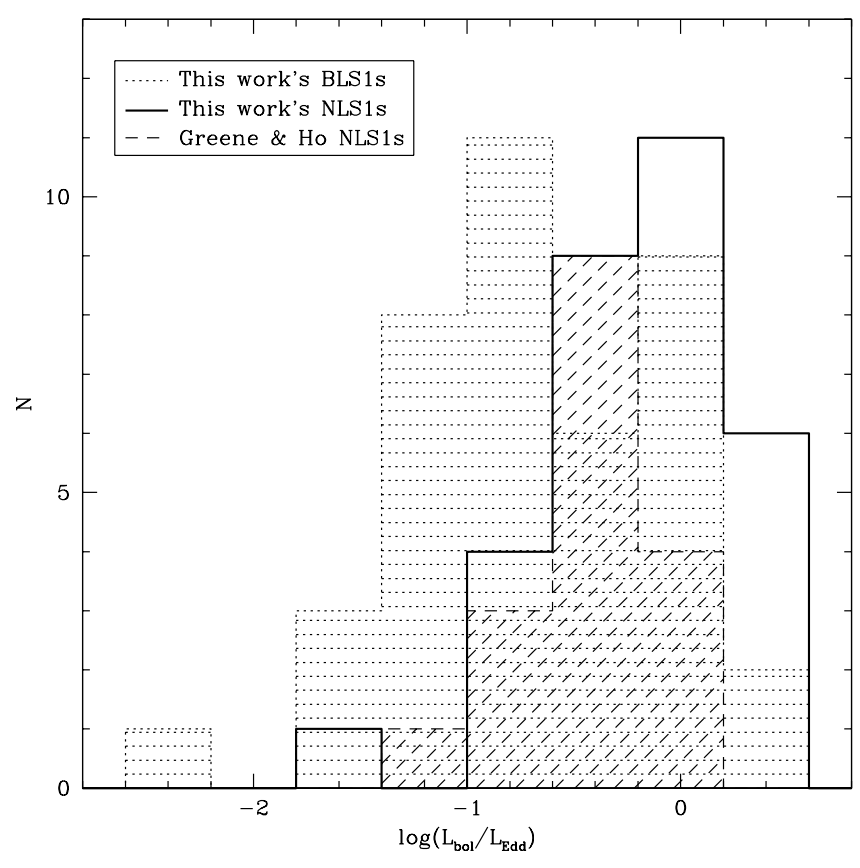

FIG. 4.-Distributions of $L_{\mathrm{bol}} / L_{\mathrm{Edd}}$ for three samples: the soft X-ray-selected BLS1s (dotted histogram) and NLS1s (solid line) from our sample, with black hole mass calculated using the $\mathrm{H} \beta$ line dispersion, and the optically selected NLS1s from Greene \& Ho (2004; dashed histogram), with black hole masses calculated using the $\mathrm{H} \alpha$ FWHM.

Ho (2004) use $L_{\text {bol }}=9.8 \lambda L_{5100}$, while Grupe et al. (2004) use the spectral energy distribution to estimate the bolometric luminosity of each AGN in this work. While the comparisons of this figure are suspect, we present it here to show the line dispersion analog of Figure 3 of Mathur \& Grupe (2005b), where the H $\beta$ FWHM is used to calculate black hole masses for the soft X-ray-selected NLS1s and BLS1s. Mathur \& Grupe (2005b) found that the soft $\mathrm{X}$-ray-selected NLS1s peak at the highest Eddington ratio [mean $\left.\log \left(L_{\text {bol }} / L_{\text {Edd }}\right)=+0.24\right]$, the optically selected NLS1s peak at a lower Eddington ratio [mean $\left.\log \left(L_{\mathrm{bol}} / L_{\mathrm{Edd}}\right)=-0.45\right]$, and the soft X-ray-selected BLS1s peak at an even lower Eddington ratio [mean $\left.\log \left(L_{\mathrm{bol}} / L_{\mathrm{Edd}}\right)=-0.75\right]$. When the line dispersion is used to calculate black hole masses for the soft X-ray-selected sample, we see that the soft X-ray-selected NLS1s have a mean $\log \left(L_{\mathrm{bol}} / L_{\mathrm{Edd}}\right)=-0.19$, while the soft X-ray-selected BLS1s have a mean $\log \left(L_{\mathrm{bol}} / L_{\mathrm{Edd}}\right)=-0.69$. The trend of soft X-rayselected NLS1s peaking at the highest Eddington ratio, the optically selected NLS1s peaking at a lower Eddington ratio, and the soft X-ray-selected BLS1s peaking at an even lower Eddington ratio remains. However, the soft X-ray-selected NLS1s have significantly lower Eddington ratios when the line dispersion rather than the FWHM is used to calculate black hole masses, and thus the trend is less pronounced. Because we have forced the BLS1s to lie near the $M_{\mathrm{BH}}-\sigma_{*}$ relation, the BLS1s peak at similar Eddington ratios for the FWHM and line dispersion cases. We cannot say where the optically selected NLS1s would lie if those black hole masses were based on the line dispersion rather than the FWHM, but perhaps they too would be shifted toward lower Eddington ratios.

\section{DISCUSSION AND CONCLUSIONS}

To address whether the results of Grupe \& Mathur (2004) are affected by substituting the $\mathrm{H} \beta$ line dispersion for the FWHM as a measure of the BLR gas velocity, we measured line dispersions and calculated line dispersion-based virial products for 71 out of the 75 AGNs studied in Grupe \& Mathur (2004). While the distributions of NLS1 and BLS1 virial products did become significantly more similar, they remain statistically distinct, with NLS1s having smaller virial products than BLS1s.

To examine the location of our AGNs on the $M_{\mathrm{BH}}-\sigma_{*}$ plane, we scaled our virial products to black hole masses using the scale factor that minimizes the rms scatter of the BLS1s around the Tremaine et al. (2002) fit to the $M_{\mathrm{BH}}-\sigma_{*}$ relation. In addition, we used $\sigma_{[\mathrm{O} \mathrm{III}]}$ as a surrogate for $\sigma_{*}$. We found that using the line dispersion to calculate black hole masses makes the NLS1 and BLS1 distributions significantly more similar in their locations on the $M_{\mathrm{BH}}-\sigma_{*}$ plane. Both our Mann-Whitney $U$-test and our rms scatter test showed that NLS1s lie below the $M_{\mathrm{BH}^{-}} \sigma_{*}$ relation when either the FWHM or the line dispersion is used to calculate black hole mass. But the result is less significant when one uses the line dispersion. Furthermore, the on average larger line dispersionbased black hole masses for our sample of NLS1s leads to a lower average Eddington ratio compared to the ratio found when FWHM is used to calculate black hole masses.

Our results are similar to those of Collin et al. (2006). They found that NLS1s and BLS1s require distinct scale factors to shift them onto the quiescent galaxy $M_{\mathrm{BH}}-\sigma_{*}$ relation when virial products are calculated using the $\mathrm{H} \beta$ FWHM. When the $\mathrm{H} \beta$ line dispersion is used, a constant scale factor is sufficient to shift both NLS1s and BLS1s onto the $M_{\mathrm{BH}}-\sigma_{*}$ relation. In this work, we approach the problem by assuming a constant scale factor whether FWHM or line dispersion is employed. Grupe \& Mathur (2004) found that many of their soft X-ray-selected NLS1s lie below the $M_{\mathrm{BH}}-\sigma_{*}$ relation when virial products are calculated using FWHM. The authors concluded that these were highly accreting NLS1s that had not yet achieved their "final" mass. In this work, we find that using the line dispersion still leaves NLS1s as a class below the $M_{\mathrm{BH}}-\sigma_{*}$ relation, but to a less significant degree than when FWHM is used.

Furthermore, we agree with Collin et al. (2006) in that FWHM is more sensitive to some physical property of the AGN, be it perhaps the Eddington ratio or inclination. In addition, the NLS1s of our sample are most affected by changing our width measure, and therefore the mystery physical property is likely enhanced in NLS1s. We note here that the Collin et al. (2006) scale factor derived to best scale line dispersion-based virial products into black hole masses $(f=3.85)$ overpredicts the majority of our BLS1 black hole masses with respect to the Tremaine et al. (2002) fit to the $M_{\mathrm{BH}}-\sigma_{*}$ relation. Even the Collin et al. scale factor computed using only their Population B broad-line objects $(f=3.75)$ is significantly larger than our value of $f=2.19$. However, the Collin et al. scale factor derived from FWHM measurements on broadline objects $(f=0.52)$ is in good agreement with our value of $f=0.53$. The fact that our FWHM scale factor is consistent with the Collin et al. value while our line dispersion scale factor is not could be due to different selection effects in the optical and soft $\mathrm{X}$-ray-selected samples. If this is the case, it could be giving us a clue about the physical property that differentiates between FWHM and line dispersion. Because of the uncertainty involved in applying the scale factor, we trust our results comparing NLS1 and BLS1 virial products more than the result comparing the loci of NLS1s and BLS1s on the $M_{\mathrm{BH}}-\sigma_{*}$ plane.

We briefly highlight the differences between reverberationmapping width measures and single-epoch width measures. In our sample, the fractional measurement errors of both the FWHM and the line dispersion are about the same $(\sim 0.05)$. Furthermore, the fractional measurement error is essentially indifferent to whether an object is a NLS1 or a BLS1. However, we could still be introducing a systematic bias into the measurement of the 
FWHM or the line dispersion by using single-epoch observations. Peterson et al. (2004) showed that, in reverberation mapping, one should measure the width of the emission line on the rms spectrum, which leaves only the variable part of the spectrum. Since we are using single-epoch observations, we must remove or avoid the constant aspects of the spectrum. For example, Grupe et al. (2004) subtracted the $\mathrm{H} \beta$ narrow component for each AGN in our sample. Because the subtraction mainly affects the core of the emission line, it primarily introduces error into our FWHM measurement that would not be present in reverberation mapping. There are also many nonvariable contaminating features surrounding $\mathrm{H} \beta$, such as [O III] and occasionally an optically thin, very broad $\mathrm{H} \beta$ component. These aspects of the spectrum mainly affect the wings of the emission line and therefore primarily introduce an error into the line dispersion that would not be present in reverberation mapping. These and other errors could mean that the width measure that is best for reverberation mapping may not be the best for single-epoch observations. Using these data, we cannot say which width measure is the "right" one. The evidence in favor of the line dispersion being the better choice is presented in Peterson et al. (2004).

In summary, for our soft X-ray-selected sample, the virial product distributions of NLS1s and BLS1s remain distinct when the line dispersion is used to measure the $\mathrm{H} \beta$ line width; the difference, however, is less significant than in the FWHM case. Similarly, on the $M_{\mathrm{BH}}-\sigma_{*}$ plane, our sample of NLS1s is shifted toward the BLS1s when the line dispersion rather than the FWHM is used; however, the NLS1s remain below the $M_{\mathrm{BH}}-\sigma_{*}$ relation. The disparity between the FWHM and line dispersion results and the level of significance of the line dispersion results are such that we cannot draw firm conclusions on the location of soft X-rayselected NLS1s on the $M_{\mathrm{BH}}-\sigma_{*}$ plane. If the scale factor to convert virial products into black hole masses is the same for NLS1s and BLS1s, we are still left with two alternative scenarios (discussed in Mathur \& Grupe 2005b), and the present data do not allow us choose one over the other. Either (1) NLS1s lie below the $M_{\mathrm{BH}}-\sigma_{*}$ relation, indicating that their black hole masses are growing, or (2) NLS1s lie on the $M_{\mathrm{BH}}-\sigma_{*}$ relation, so they preferentially reside in smaller mass, less luminous galaxies. In the end, more trustworthy stellar velocity dispersions and accurate black hole mass measurements with reverberation mapping are required for a firmer statement about the locus of NLS1s on the $M_{\mathrm{BH}}-\sigma_{*}$ plane. Even more basic, we must securely determine which physical property of AGNs distinguishes FWHM from line dispersion as a BLR gas velocity measure.

We would like to thank Bradley Peterson for helpful discussions and for generously allowing us to use his program to calculate line dispersions. We also thank the anonymous referee for useful comments. L. W. is supported by a Graduate Fellowship from the National Science Foundation.
Barth, A., Greene, J., \& Ho, L. 2005, ApJ, 619, L151

Bentz, M. C., Peterson, B. M., Pogge, R. W., Vestergaard, M., \& Onken, C. A. 2006, ApJ, 644, 133

Blandford, R. D., \& McKee, C. F. 1982, ApJ, 255, 419

Boroson, T. A. 2003, ApJ, 585, 647

Collin, S., Kawaguchi, T., Peterson, B. M., \& Vestergaard, M. 2006, A\&A, 456, 75

Ferrarese, L., \& Ford, H. 2005, Space Sci. Rev., 116, 523

Ferrarese, L., \& Merritt, D. 2000, ApJ, 539, L9

Ferrarese, L., Pogge, R. W., Peterson, B. M., Merritt, D., Wandel, A., \& Joseph, C. L. 2001, ApJ, 555, L79

Gebhardt, K., et al. 2000a, ApJ, 539, L13 2000b, ApJ, 543, L5

Genzel, R., Pichon, C., Eckart, A., Gerhard, O. E., \& Ott, T. 2000, MNRAS, 317,348

Ghez, A. M., Morris, M., Becklin, E. E., Tanner, A., \& Kremenek, T. 2000, Nature, 407, 349

Greene, J. E., \& Ho, L. C. 2004, ApJ, 610, 722

2005, ApJ, 627, 721

Grupe, D., Beuermann, K., Thomas, H.-C., Mannheim, K., \& Fink, H. H. 1998, A\&A, 330, 25

\section{REFERENCES}

Grupe, D., \& Mathur, S. 2004, ApJ, 606, L41

Grupe, D., Wills, B. J., Leighly, K. M., \& Meusinger, H. 2004, AJ, 127, 156

Kaspi, S., Smith, P. S., Netzer, H., Maoz, D., Jannuzi, B. T., \& Giveon, U. 2000, ApJ, 533, 631

Mathur, S., \& Grupe, D. 2005a, A\&A, 432, 463

2005b, ApJ, 633, 688

Mathur, S., Kuraszkiewicz, J., \& Czerny, B. 2001, NewA, 6, 321

Miyoshi, M., Moran, J., Herrnstein, J., Greenhill, L., Nakal, N., Diamond, P., \& Inoue, M. 1995, Nature, 373, 127

Onken, C. A., Ferrarese, L., Merritt, D., Peterson, B. M., Pogge, R. W., Vestergaard, M., \& Wandel, A. 2004, ApJ, 615, 645

Osterbrock, D. E., \& Pogge, R. W. 1985, ApJ, 297, 166

Peterson, B. M. 1993, PASP, 105, 247

Peterson, B. M., et al. 2004, ApJ, 613, 682

Pounds, K. A., Done, C., \& Osborne, J. P. 1995, MNRAS, 277, L5

Press, W. H., Teukolsky, S. A., Vetterling, W. T., \& Flannery, B. P. 1992, Numerical Recipes in C: The Art of Scientific Computing (2nd ed.; Cambridge: Cambridge Univ. Press)

Shields, J. C., Ferland, G. J., \& Peterson, B. M. 1995, ApJ, 441, 507

Tremaine, S., et al. 2002, ApJ, 574, 740 